\title{
Case study of thrombosis and thrombocytopenia syndrome following administration of the AstraZeneca COVID-19 vaccine
}

Ashraf Saleh, Joel Collins

\section{CASE}

A woman aged 63 years presented to a local general practice with a 24-hour history of persistent headache, listlessness, anorexia and nausea. She was uncharacteristically lying down in the clinic and complaining of intractable headache and malaise. She had no medical history apart from quiescent Hashimoto's thyroiditis, for which she took $75 \mu \mathrm{g}$ levothyroxine. She had no known medication allergies and no history of venous thromboembolism. A first AstraZeneca COVID-19 vaccine had been administered one week prior.

She was systemically well, with blood pressure 125/75 $\mathrm{mmHg}$ while lying down and a regular pulse rate of 78 beats per minute, a respiratory rate of 16 breaths per minute and oxygen saturation of $98 \%$ on room air. She had a clear ear, nose and throat examination. Cardiovascular examination results were normal. Heart sounds were dual with no murmurs. She remained neurologically intact throughout the examination, with a Glascow Coma Scale score of 15 . She responded partially to $5 \mathrm{mg}$ of morphine given intramuscularly while awaiting urgent blood tests.

\section{Findings}

The initial full blood examination revealed a platelet count of $116 \times 10^{9} / \mathrm{L}$, which was significantly reduced from her usual baseline $\left(>320 \times 10^{9} / \mathrm{L}\right.$; reference range $\left.150-400 \times 10^{9} / \mathrm{L}\right)$. Her initial international normalised ratio was 1.4 (reference range $<1.3$ ), with a normal fibrinogen level of $2.59 \mathrm{~g} / \mathrm{L}$ (reference range 1.89-4.20 g/L) and elevated D-dimer of $14.19 \mu \mathrm{g} / \mathrm{mL}$ fibrinogen equivalent units ( $>35$ times upper limit of normal). Cerebral venography by computed tomography was arranged, showing a left sigmoid sinus thrombosis extending to the left internal jugular vein with components within the left transverse sinus.

\section{QUESTION 1}

How does thrombosis and

thrombocytopenia syndrome (TTS) develop?

\section{QUESTION 2}

What is the epidemiology of TTS?

ANSWER 1

Vaccine-induced immune thrombotic thrombocytopenia appears to be caused by circulating immunoglobulin $\mathrm{G}$ antibodies with affinity for platelet factor 4 (PF4) expressed on platelets. These antibodies are thought to activate platelets and promote clot formation (and platelet consumption) via binding to Fc $\gamma$ IIA receptors. ${ }^{1}$ This phenomenon has been reported following administration of both the ChAdOx1 nCoV-19 vaccine (AstraZeneca, University of Oxford) ${ }^{2}$ and the Ad26.COV2.S vaccine (Janssen, Johnson \& Johnson). ${ }^{3}$ In comparison to typical venous thromboembolism, TTS-related thrombosis is more commonly detected in atypical sites including the splanchnic, adrenal, cerebral and ophthalmic veins. Both venous and arterial thrombosis are reported.

\section{ANSWER 2}

Australia has recorded 151 confirmed or probable cases of TTS and eight fatalities following 12 million doses of the ChAdOx1 nCoV-19 vaccine, with an incidence of 2.0 TTS cases per 100,000 vaccine doses as of 12 August $2021 .^{4}$

Cerebral venous sinus thrombosis (CVST) also occurs in the setting of actual COVID-19 infection. An Oxford retrospective study indicated that the incidence of CVST is significantly increased in the event of COVID-19 infection (42.8 per million COVID-19 cases) when compared with ChAdOx1 nCoV-19 vaccination. ${ }^{5}$

\section{CASE CONTINUED}

The patient was admitted to hospital directly from the general practice, and 
consultation with a haematologist was sought. The patient was subsequently administered a non-heparin based anticoagulant (rivaroxaban $15 \mathrm{mg}$ twice daily) and intravenous immunoglobulin therapy (Intragam) at $1 \mathrm{~g} / \mathrm{kg}$ bodyweight daily for two days as per CSL Behring protocol. Her platelet count remained stable at $100 \times 10^{9} / \mathrm{L}$ for the next 48 hours, then rose to $135 \times 10^{9} / \mathrm{L}$ at 72 hours, $180 \times 10^{9} / \mathrm{L}$ at four days and $216 \times 10^{9} / \mathrm{L}$ at five days after commencement of Intragam. The patient was then discharged with simple analgesia and instructions to continue direct oral anticoagulant therapy for six months. She underwent weekly full blood examinations to monitor platelet counts for recurrence as the infused intravenous immunoglobulins are depleted over the subsequent 14-21 days.

Ultimately, her heparin/PF4 antibody enzyme-linked immunosorbent assay and functional assay were both positive, confirming the diagnosis.

Three weeks later, the patient's platelet count reached her normal baseline level of $300 \times 10^{9} / \mathrm{L}$, and the nausea and headache she previously experienced subsided. She was able to return to her workplace within four weeks.

\section{QUESTION 3}

What should clinicians look for in assessing CVST?

\section{ANSWER 3}

CVST is a recognised complication of the ChAdOx1 nCoV-19 vaccine that should elicit a high index of clinical suspicion when patients of any age present with persistent headache $4-42$ days post administration of the vaccine.

All patients with symptoms consistent with CVST (persistent, severe and exacerbated by recumbency and Valsalva) within the relevant time criteria should be assessed initially by blood investigation assessing for (relative) thrombocytopenia and elevated $\mathrm{D}$-dimer ( $>5$ times the upper limit of normal ${ }^{6}$ ), and subsequently by imaging if this blood screening yields positive results. Consideration for haematological advice should be sought early for possible TTS cases.

It is important that all general practitioners are aware of the signs/ symptoms of CVST and other thrombotic complications of the ChAdOx $1 \mathrm{nCoV}-19$ vaccine and have a low threshold for consideration of an investigation of this diagnosis.

First published 12 November 2021.

\section{Authors}

Ashraf Saleh MBBS, MNutrSci, BMedSci, FRACGP, FARGP (Emergency Medicine), Senior Medical Officer, Toowoomba Private Emergency Group, St Vincent's Toowoomba Private Hospital, Toowoomba, Qld; General Practitioner, Rangeside Medical Centre, Toowoomba, Qld

Joel Collins MBBS, FRACP, Practice Principal, Toowoomba Haematology, Toowoomba, Qld; Staff Specialist, Toowoomba Base Hospital, Toowoomba, Qld Competing interests: None.

Funding: None.

Provenance and peer review: Not commissioned, peer reviewed.

Citation: Saleh A, Collins J. Case study of thrombosis and thrombocytopenia syndrome following administration of the AstraZeneca COVID-19 vaccine. Aust J Gen Pract 2021;50 Suppl 52. doi: 10.31128/

AJGP-COVID-52

Correspondence to:

joel@drjoelcollins.com

\section{References}

1. Flannagan RS, Jaumouillé V, Grinstein S. The cell biology of phagocytosis. Annu Rev Pathol 2012;7:61-98. doi: 10.1146/annurevpathol-011811-132445

2. Greinacher A, Thiele T, Warkentin TE, Weisser K, Kyrle PA, Eichinger S. Thrombotic thrombocytopenia after ChAdOx1 nCov-19 vaccination. N Engl J Med 2021;384(22):2092-101. doi: 10.1056/NEJMoa2104840.

3. Muir KL, Kallam A, Koepsell SA, Gundabolu K. Thrombotic thrombocytopenia after Ad26.COV2.S vaccination. N Engl J Med 2021;384(20):1964-65. doi: 10.1056/NEJMc2105869.

4. Therapeutic Goods Administration. COVID19 vaccine weekly safety report 28-08-2021. Canberra, ACT: DoH Therapeutic Goods Administration, 2021. Available at www.tga.gov. $\mathrm{au} /$ periodic/covid-19-vaccine-weekly-safetyreport-26-08-2021 [Accessed 23 October 2021].

5. Taquet M, Husain M, Geddes JR, Luciano S, Harrison PJ. Cerebral venous thrombosis and portal vein thrombosis: A retrospective cohort study of 537,913 COVID-19 cases. EClinicalMedicine 2021;39:101061. doi: 10.1016/j. eclinm.2021.101061.

6. Australasian College for Emergency Medicine. Assessment of patients presenting to the ED with TTS symptoms following COVID-19 vaccination. West Melbourne, Vic: ACEM, 2021. 